\title{
Avaliação da suplementação concentrada em pastagem de Tifton-85 sobre os componentes não carcaça de $\operatorname{cordeiros}^{1}$
}

\author{
Evaluation of concentrated supplementation in Tifton-85 pasture \\ on the components other then carcass of lambs
}

\section{Sérgio Carvalho ${ }^{2}$ André Vergueiro ${ }^{3}$ Roberto Kieling ${ }^{2}$ Regina Cánovas Teixeira ${ }^{2}$ Josane Pivato $^{4}$ Renan Viero $^{5}$ Alex Nunez da Cruz ${ }^{6}$}

\section{RESUMO}

Este estudo objetivou realizar uma análise dos componentes não carcaça ("quinto quarto") de cordeiros submetidos a diferentes níveis de suplementação concentrada em pastagem de Tifton-85. Utilizaram-se 25 cordeiros, machos castrados, sem raça definida, os quais receberam em condição de pastejo, diferentes níveis de suplementação: 0\%, 1,0\%, 1,5\%, 2,0\% ou $2,5 \%$ do peso vivo. $O$ alimento concentrado utilizado foi fornecido pela empresa BÜNGE alimentos, ração Sano Vitosan, com o nome comercial de ovino criador. Após cada abate, os componentes corporais dos animais foram pesados individualmente. Posteriormente, calculou-se a percentagem dos componentes corporais em relação ao peso vivo. Os pesos de sangue, pele, patas, coração, pulmão + traquéia e de gordura interna aumentaram linearmente com o aumento do nível de suplemento concentrado oferecido. Por outro lado, verificou-se efeito linear decrescente do nível de suplementação sobre as proporções de cabeça e de rins. Os valores médios obtidos foram: sangue $=1,208 \mathrm{~kg}$ e 3,68\%; pele $=2,958 \mathrm{~kg}$ e 8,98\%; patas $=$ $0,643 \mathrm{~kg}$ e 1,97\%; cabeça $=1,184 \mathrm{~kg}$ e 3,64\%; coração $=0,124 \mathrm{~kg}$ e 0,38\%; rins $=0,088 \mathrm{~kg}$ e 0,27\%; fígado $=0,507 \mathrm{~kg}$ e $1,55 \%$; pulmão + traquéia $=0,573 \mathrm{~kg}$ e 1,75\%; baço $=0,047 \mathrm{~kg}$ e 0,14\%; gordura interna $=0,202 \mathrm{~kg}$ e $0,61 \%$ e gordura renal $=0,150 \mathrm{~kg} e$ $0,46 \%$. Os pesos de vísceras verdes cheias e de conteúdo gastrintestinal, quando expressos em $\mathrm{kg}$, as proporções de vísceras verdes cheias, vísceras verdes vazias e de conteúdo gastrintestinal diminuíram linearmente com o aumento do nível de suplementação concentrada. Os valores médios verificados foram: vísceras verdes cheias $=8,42 \mathrm{~kg}$ e $25,99 \%$; vísceras verdes vazias $=2,71 \mathrm{~kg}$ e $8,29 \%$ e conteúdo gastrintestinal $=5,71 \mathrm{~kg}$ e $17,69 \%$. $\begin{aligned} \text { Palavras-chave: } & \text { ovinos, produção animal, suplementação, } \\ & \text { vísceras. }\end{aligned}$

\section{ABSTRACT}

This study aimed to perform an analysis of the weight of live components ("fifth quarter") of lambs subjected to different levels of concentrated supplementation in Tifton-85 pasture. Twenty-five lambs were used, all of them castrated males, of no specific breed, all of which received in pasture conditions, different levels of supplementation: $0 \%, 1.0 \%, 1.5 \%, 2.0 \%$ or $2.5 \%$ of the living weight. The Food Company BÜNGE supplied the concentrated food used, ration Sano Vitosan, with the market name of ovine breeder. After each slaughtering the body parts of the animals were individually weighed. After that, the percentage of the body parts in relation to the living weight was calculated. The weights of blood, skin, foot, heart, lungs + trachea and inner fat have increased linearly with the increasing of the level of concentrated supplement, which was offered. On the other hand, a linear decreasing effect of the level of supplementation on the proportions of head and kidney was observed. The average amounts obtained were: blood $=1.208 \mathrm{~kg}$ and $3.68 \%$; skin $=$ $2.958 \mathrm{~kg}$ and $8.98 \%$; foot $=0.643 \mathrm{~kg}$ and $1.97 \%$; head $=1.184 \mathrm{~kg}$ and $3.64 \%$; heart $=0.124 \mathrm{~kg}$ and $0.38 \% ;$ kidney $=0.088 \mathrm{~kg}$ and $0.27 \%$; liver $=0.507 \mathrm{~kg}$ and $1.55 \% ;$ lung + trachea $=0.573 \mathrm{~kg}$ and $1.75 \%$; dull $=0.047 \mathrm{~kg}$ and $0.14 \%$; inner fat $=0.202 \mathrm{~kg}$ and $0.61 \%$ and kidney fat $=0.150 \mathrm{~kg}$ and $0.46 \%$. The weights of full green viscera and the gastrointestinal content, when expressed in $\mathrm{kg}$, and the proportions of full green viscera, empty green viscera and the gastrointestinal content declined linearly with the increasing of the level of concentrated supplementation. The

'Parte do projeto de pesquisa "Desenvolvimento da Ovinocultura na Região do Vale do Rio do Sinos".

${ }^{2}$ Professor do curso de Engenharia de Produção (Habilitação Agroindustrial) e Coordenador do Grupo de Pesquisa em Agronegócios, Centro Universitário Feevale, RS, 239, 2755, 93510-250, Novo Hamburgo, RS. E-mail: sergiocarvalho@ feevale.br (Autor para correspondência).

${ }^{3}$ Zootecnista, Técnico da Empresa BÜNGE Alimentos SA. E-mail: andre.vergueiro@bunge.com.

${ }^{4}$ Aluno de graduação do curso de Engenharia de Produção (Habilitação Agroindustrial) e Bolsista de Iniciação Científica do Grupo de

Estudos em Agronegócios do Centro Universitário Feevale.

${ }^{5}$ Aluno de graduação do curso de Engenharia de Produção (Habilitação Agroindustrial).

${ }^{6}$ Aluno de graduação do curso de Biologia do Centro Universitário Feevale. 
average amounts obtained were: full green viscera $=8.42$ and $25.99 \%$; empty green viscera $=2.71$ and $8.29 \%$ and gastrointestinal content $=5,71$ and $17,69 \%$.

Key words: animal production, sheep, supplementation, visceras.

\section{INTRODUÇÃO}

Recentemente, tem se verificado um grande número de pesquisas com o intuito de investigar a produção de carne ovina. Contudo, poucos trabalhos têm sido conduzidos objetivando realizar uma avaliação dos componentes não carcaça que fazem parte do corpo do animal. Segundo OLIVEIRA et al. (2002), provavelmente, este reduzido interesse se deva ao fato de estas variáveis não fazerem parte da carcaça comercial.

De acordo com OSÓRIO (1992), os componentes não carcaça, também denominados como "quinto quarto", representam todos os componentes do peso vivo (sistema digestório e seu conteúdo, sangue, pele, cabeça, patas, pulmão com traquéia, fígado, coração, rins, baço, gorduras interna e renal e testículos) excetuando-se a carcaça. Convém ressaltar que o peso relativo dos componentes não carcaça pode variar de 40 a $60 \%$ do peso vivo, sendo influenciado pela genética, idade, peso vivo, sexo, tipo de nascimento e alimentação.

É importante enfatizar que, na cadeia produtiva da ovinocultura, todos os segmentos, desde o produtor até o consumidor, têm uma finalidade lucrativa. Nesse sentido, para que haja uma melhor remuneração e valorização da produção ovina, a comercialização deveria ser feita considerando o animal como um todo, remunerando além da carcaça, os componentes não carcaça (OSÓRIO \& ASTIZ, 1996).

Para OSÓRIO et al. (1996a), o não aproveitamento adequado dos componentes do quinto quarto representa uma perda de alimento e matéria prima que poderiam colaborar para diminuir o preço dos produtos para os consumidores e melhorar o nível de vida das populações de baixo poder aquisitivo. Além disso, outro aspecto importante a ser considerado é que a valorização dos componentes não carcaça motivará o produtor a ter maiores cuidados sanitários, desinfetando as vísceras, melhorando, assim, as condições para que o animal manifeste seu potencial genético.

Outro fator importante a ser salientado é a estreita relação que o peso dos componentes do "quinto quarto" apresentam em relação ao rendimento de carcaça. Neste sentido, SIQUEIRA et al. (2001) descrevem que, entre os componentes não carcaça, a pele e o trato gastrintestinal cheio podem representar até mais do que $25 \%$ do peso vivo ao abate e, portanto, afetar de forma significativa o rendimento.

Com isso, o objetivo deste trabalho foi verificar o efeito de cinco níveis de suplementação concentrada sobre os componentes não carcaça de cordeiros terminados em pastagem de Tifton-85.

\section{MATERIAL E MÉTODOS}

O experimento foi conduzido em uma propriedade rural localizada no município de Portão, RS, no período de 23 de setembro a 19 de dezembro de 2003. O trabalho foi desenvolvido mediante parceria entre o Centro Universitário Feevale e a empresa BÜNGE Alimentos.

Foram utilizados 25 cordeiros de parto simples, sem raça definida, os quais foram divididos em cinco tratamentos compostos por níveis de suplementação concentrada em diferentes percentagens do peso vivo: $0 \% ; 1,0 \%, 1,5 \%, 2,0 \%$ ou $2,5 \%$. Quando os cordeiros atingiram em média 90 dias de idade, os mesmos foram desmamados e distribuídos em uma área de pastagem de Tifton-85, subdividida com cerca elétrica, em cinco piquetes medindo 16 x 68 metros, onde recebiam o suplemento de acordo com o tratamento. O período experimental foi precedido por um período pré-experimental de 14 dias que serviu para adaptar os animais ao desmame, ao alimento concentrado e ao manejo.

Os animais permaneceram por 24 horas em condição de pastejo, sendo que a oferta diária de concentrado foi dividida em duas vezes. Os horários de oferta do suplemento foram às 8:00 e 17:00 horas. A quantidade de concentrado a ser oferecida como suplemento aos animais foi ajustada diariamente, tomando como base o ganho de peso médio diário do cordeiro no período anterior. O concentrado utilizado na suplementação dos cordeiros foi fornecido pela empresa BÜNGE Alimentos, ração Sano Vitosan, com nome comercial de Ovino Criador. Em todos os tratamentos encontrava-se água e sal comum à vontade para os animais.

Todos os cordeiros foram pesados no início e ao final do período experimental, sendo que, para um melhor acompanhamento do desempenho, foram realizadas pesagens intermediárias. Os animais foram dosificados com vermífugo para controle de endoparasitos no início do experimento e a cada 30 dias. Os cordeiros foram avaliados por um período de 73 dias, quando então foram abatidos.

O abate ocorreu após jejum de sólidos de 14 horas, sendo que, neste momento, os animais foram

Ciência Rural, v.35, n.2, mar-abr, 2005. 
pesados obtendo-se o peso vivo médio ao abate. O sangue foi coletado em balde plástico previamente tarado e pesado. Em seguida, procedeu-se a evisceração, sendo os componentes corporais pesados individualmente. Assim, determinaram-se individualmente os pesos de pele, patas, cabeça, coração, rins, fígado, pulmão com traquéia, baço, gordura interna e gordura renal. $\mathrm{O}$ trato gastrintestinal foi pesado cheio e, em seguida, procederam-se esvaziamento e minuciosa lavagem. Após ter escorrido a água, procedeu-se nova pesagem e, por diferença, obteve-se o conteúdo gastrintestinal. Posteriormente, calculou-se a percentagem dos componentes corporais em relação ao peso vivo no momento do abate.

$\mathrm{O}$ delineamento experimental utilizado foi o inteiramente casualizado, com cinco tratamentos e cinco repetições. Os dados foram avaliados por meio de análise de variância e de regressão, utilizando-se o pacote estatístico SAS (1993), adotando-se um nível de significância de $5 \%$.

\section{RESULTADOS E DISCUSSÃO}

Os valores médios correspondentes aos componentes do peso vivo avaliados, expressos em $\mathrm{kg}$ e em porcentagem do peso vivo, são apresentados nas tabelas 1 e 2, respectivamente. Verificou-se efeito do nível de suplementação sobre os pesos de sangue, pele, patas, coração, pulmão + traquéia e gordura interna, os quais aumentaram linearmente com a elevação do nível de concentrado oferecido na forma de suplemento. Este efeito pode ser explicado pelo peso vivo ao abate dos cordeiros, o qual se elevou à medida que se incrementou o nível de suplementação. Com uma maior velocidade de crescimento dos cordeiros, houve um acompanhamento no crescimento em termos absolutos dos componentes corporais avaliados. Contudo, quando se analisa em termos percentuais (Tabela 2), verifica-se que não houve efeito do nível de suplementação em relação a estes componentes corporais.

Com relação às proporções de cabeça e de rins, verifica-se que as mesmas diminuíram linearmente com a elevação do nível de suplementação (Tabela 2). Uma vez que em termos absolutos houve uma similaridade destes dois componentes corporais entre os tratamentos (Tabela 1), quando se avaliou proporcionalmente em relação ao peso vivo, ocorreu diminuição da proporção dos componentes em detrimento do aumento do peso vivo verificado com o incremento do nível de suplementação.

Os resultados obtidos no presente estudo são concordantes com OSÓRIO et al. (1996b), os quais avaliaram os componentes corporais de cordeiros da raça Corriedale abatidos com $30,63 \mathrm{~kg}$ de peso vivo e encontraram os valores de $1,97 \%$ para patas, $4,03 \%$ para cabeça, $0,44 \%$ para coração, $0,25 \%$ para rins, $1,38 \%$ para fígado, $1,69 \%$ para pulmão + traquéia e $0,13 \%$ para baço. Contudo, os valores obtidos por esses autores de $11,96 \%$ para pele ficam acima do observado no presente estudo. Esta diferença pode ser explicada pelo fato de que estes autores trabalharam

Tabela 1 - Médias, coeficientes de variação (CV) e equações de regressão ajustadas (ER), para peso vivo ao abate e para os componentes do peso vivo, em $\mathrm{kg}$, de acordo com os tratamentos.

\begin{tabular}{|c|c|c|c|c|c|c|c|}
\hline \multirow{2}{*}{ Itens } & \multicolumn{5}{|c|}{ Nível de suplementação (\% do peso vivo) } & \multirow{2}{*}{$\mathrm{CV}(\%)$} & \multirow{2}{*}{ ER } \\
\hline & 0 & 1,0 & 1,5 & 2,0 & 2,5 & & \\
\hline Peso vivo ao abate & 28,84 & 31,54 & 33,92 & 34,20 & 35,52 & 11,86 & 1 \\
\hline Sangue & 1,028 & 1,157 & 1,341 & 1,188 & 1,326 & 15,54 & 2 \\
\hline Pele & 2,529 & 2,964 & 2,910 & 3,246 & 3,143 & 18,25 & 3 \\
\hline Patas & 0,594 & 0,598 & 0,678 & 0,668 & 0,678 & 12,37 & 4 \\
\hline Cabeça & 1,116 & 1,162 & 1,267 & 1,183 & 1,194 & 8,79 & $\mathrm{Y}=1,184$ \\
\hline Coração & 0,116 & 0,117 & 0,132 & 0,119 & 0,138 & 11,99 & 5 \\
\hline Rins & 0,082 & 0,085 & 0,099 & 0,083 & 0,090 & 15,25 & $\mathrm{Y}=0,088$ \\
\hline Fígado & 0,443 & 0,512 & 0,546 & 0,476 & 0,560 & 15,57 & $Y=0,507$ \\
\hline Pulmão + Traquéia & 0,500 & 0,557 & 0,611 & 0,600 & 0,597 & 13,43 & 6 \\
\hline Baço & 0,040 & 0,050 & 0,049 & 0,043 & 0,053 & 18,84 & $Y=0,047$ \\
\hline Gordura interna & 0,142 & 0,217 & 0,155 & 0,232 & 0,263 & 39,37 & 7 \\
\hline Gordura renal & 0,136 & 0,189 & 0,149 & 0,148 & 0,128 & 52,56 & $Y=0,150$ \\
\hline
\end{tabular}

$1 . \mathrm{Y}=29,02324+0,27005 * \mathrm{SUP}, \mathrm{R}^{2}=0,28 ; 2 . \mathrm{Y}=1,05532+0,01091 * \mathrm{SUP}, \mathrm{R}^{2}=0,21 ; 3 . \mathrm{Y}=2,59149+0,02621 * \mathrm{SUP}, \mathrm{R}^{2}=0,16 ; 4 . \mathrm{Y}=$ $0,58886+0,00388 * \mathrm{SUP}, \mathrm{R}^{2}=0,16 ; 5 . \mathrm{Y}=0,11411+0,00073 * \mathrm{SUP}, \mathrm{R}^{2}=0,16 ; 6 . \mathrm{Y}=0,51435+0,00419 * \mathrm{SUP}, \mathrm{R}^{2}=0,19 ; 7 . \mathrm{Y}=0,14186+$ $0,00428 *$ SUP $, R^{2}=0,19$

* e ** significativo a 5 e $1 \%$ de probabilidade, respectivamente, pelo teste F; SUP = nível de suplementação.

Ciência Rural, v.35, n.2, mar-abr, 2005. 
Tabela 2 - Médias, coeficientes de variação (CV) e equações de regressão ajustadas (ER), para os componentes do peso vivo, em \%, de acordo com os tratamentos.

\begin{tabular}{|c|c|c|c|c|c|c|c|}
\hline \multirow{2}{*}{ Itens } & \multicolumn{5}{|c|}{ Nível de suplementação (\% do peso vivo) } & \multirow[t]{2}{*}{$\mathrm{CV}(\%)$} & \multirow[t]{2}{*}{ ER } \\
\hline & 0 & 1,0 & 1,5 & 2,0 & 2,5 & & \\
\hline Sangue & 3,57 & 3,67 & 3,96 & 3,48 & 3,72 & 8,22 & $\mathrm{Y}=3,68$ \\
\hline Pele & 8,76 & 9,39 & 8,45 & 9,47 & 8,80 & 10,63 & $\mathrm{Y}=8,98$ \\
\hline Patas & 2,07 & 1,91 & 2,01 & 1,95 & 1,91 & 8,59 & $\mathrm{Y}=1,97$ \\
\hline Cabeça & 3,91 & 3,71 & 3,76 & 3,47 & 3,37 & 7,38 & 1 \\
\hline Coração & 0,40 & 0,38 & 0,39 & 0,35 & 0,39 & 8,01 & $\mathrm{Y}=0,38$ \\
\hline Rins & 0,29 & 0,27 & 0,29 & 0,24 & 0,26 & 10,62 & 2 \\
\hline Fígado & 1,55 & 1,62 & 1,61 & 1,40 & 1,57 & 8,00 & $\mathrm{Y}=1,55$ \\
\hline Pulmão + Traquéia & 1,73 & 1,77 & 1,82 & 1,75 & 1,68 & 8,57 & $Y=1,75$ \\
\hline Baço & 0,14 & 0,16 & 0,14 & 0,13 & 0,15 & 17,12 & $Y=0,14$ \\
\hline Gordura interna & 0,48 & 0,68 & 0,45 & 0,68 & 0,74 & 38,50 & $Y=0,61$ \\
\hline Gordura renal & 0,45 & 0,61 & 0,43 & 0,43 & 0,36 & 53,44 & $Y=0,46$ \\
\hline
\end{tabular}

$1 . \mathrm{Y}=3,94551-0,02151 * * \mathrm{SUP}, \mathrm{R}^{2}=0,34 ; 2 . \mathrm{Y}=0,28973-0,00144 * \mathrm{SUP}, \mathrm{R}^{2}=0,17$

$* \mathrm{e}^{* *}$ significativo a 5 e $1 \%$ de probabilidade, respectivamente, pelo teste $\mathrm{F} ; \mathrm{SUP}=$ nível de suplementação.

com cordeiros da raça Corriedale que apresentam uma elevada capacidade produtiva de lã, o que fez com que a proporção de pele fosse maior.

Com relação à percentagem de sangue, o valor médio obtido de 3,68\% encontra-se próximo ao verificado por SIQUEIRA et al. (2001), os quais avaliando os componentes corporais de cordeiros machos abatidos com diferentes pesos vivo, observaram um valor médio de 3,92\%.

Na tabela 3, são apresentados os valores médios para vísceras verdes cheias (VISCHE), vísceras verdes vazias (VISVAZ) e conteúdo gastrintestinal (CGI), em kg e em percentagem, de acordo com os tratamentos. Verifica-se que houve redução linear do CGI dos cordeiros, expresso em $\mathrm{kg}$, à medida que ocorreu aumento do nível de suplementação concentrada. Como conseqüência, observa-se que o peso de VISCHE também diminuiu linearmente com o aumento da suplementação. Por outro lado, não houve efeito ( $P>0,05)$ do nível de suplementação sobre o peso de VISVAZ.

O aumento do CGI verificado com a redução do nível de suplementação pode ser explicado pela necessidade dos animais em ingerir uma maior quantidade de pasto visando atender a sua necessidade energética. Segundo MERTENS (1992), alimentos volumosos apresentam uma baixa densidade energética quando comparados a alimentos concentrados, o que leva a uma maior necessidade de ingestão.

As proporções de VISCHE, VISVAZ e de CGI diminuíram linearmente $(\mathrm{P}<0,01)$ com o aumento do nível de suplementação concentrada. Este aspecto é importante do ponto de vista comercial pois, uma menor proporção de CGI e, conseqüentemente, de

Tabela 3 - Médias, coeficientes de variação (CV) e equações de regressão ajustadas (ER), para vísceras verdes cheias (VISCHE), vísceras verdes vazias (VISVAZ) e conteúdo gastrintestinal (CGI), de acordo com os tratamentos.

\begin{tabular}{|c|c|c|c|c|c|c|c|}
\hline \multirow{2}{*}{ Itens } & \multicolumn{5}{|c|}{ Nível de suplementação (\% do peso vivo) } & \multirow{2}{*}{$\mathrm{CV}(\%)$} & \multirow{2}{*}{ ER } \\
\hline & 0 & 1,0 & 1,5 & 2,0 & 2,5 & & \\
\hline VISCHE (kg) & 9,170 & 8,988 & 9,798 & 7,217 & 6,925 & 19,71 & 1 \\
\hline VISVAZ (kg) & 2,609 & 2,726 & 3,080 & 2,537 & 2,573 & 16,67 & $Y=2,71$ \\
\hline CGI (kg) & 6,561 & 6,262 & 6,718 & 4,680 & 4,352 & 22,19 & 2 \\
\hline VISCHE (\%) & 31,38 & 28,73 & 29,15 & 21,19 & 19,49 & 15,73 & 3 \\
\hline VISVAZ (\%) & 8,97 & 8,68 & 9,17 & 7,42 & 7,22 & 12,07 & 4 \\
\hline CGI (\%) & 22,40 & 20,04 & 19,97 & 13,77 & 12,27 & 18,38 & 5 \\
\hline
\end{tabular}

$1 . \mathrm{Y}=9,74608-0,09475 * \mathrm{SUP}, \mathrm{R}^{2}=0,21 ; 2 . \mathrm{Y}=7,00986-0,09252 * * \mathrm{SUP}, \mathrm{R}^{2}=0,30 ; 3 . \mathrm{Y}=32,93146-0,49608 * * \mathrm{SUP}, \mathrm{R}^{2}=0,54 ; 4 . \mathrm{Y}=$ $9,32546-0,07368 * * \mathrm{SUP}, \mathrm{R}^{2}=0,30 ; 5$. Y=23,60600-0,42240**SUP, $\mathrm{R}^{2}=0,57$.

$*$ e $* *$ significativo a 5 e $1 \%$ de probabilidade, respectivamente, pelo teste $\mathrm{F}$; SUP = nível de suplementação.

Ciência Rural, v.35, n.2, mar-abr, 2005. 
VISCHE, está associada a um melhor rendimento de carcaça, o que faz com que uma maior proporção de material comestível esteja disponível para o consumidor.

$O$ resultado obtido no presente trabalho para a proporção de VISCHE dos animais que não receberam suplementação concentrada, está de acordo com OSÓRIO et al. (1996b), os quais verificaram em cordeiros da raça Corriedale, machos castrados, criados em condição de campo nativo e abatidos com $29,57 \mathrm{~kg}$ de peso vivo, um valor médio de $30,07 \%$. Por outro lado, o valor médio verificado para proporção de CGI que foi de $17,69 \%$, está em desacordo com SIQUEIRA et al. (2001), os quais avaliando cordeiros Ile de France x Corriedale, terminados em confinamento e abatidos com peso vivo entre 28 e $40 \mathrm{~kg}$, obtiveram um valor médio de $13,4 \%$.

\section{CONCLUSÃO}

O aumento do nível de suplementação concentrada em pastagem de Tifton- 85 proporciona aumento nos pesos de sangue, pele, patas, coração, pulmão + traquéia e gordura interna. De forma inversa, as proporções de cabeça e de rins diminuíram, linearmente, com a elevação do nível de suplementação.

O aumento da suplementação concentrada, em pastagem de Tifton-85, proporciona redução linear do conteúdo gastrintestinal e das vísceras verdes cheias, quando expressos em $\mathrm{kg}$ e em porcentagem do peso vivo. Da mesma forma, a proporção de vísceras verdes vazias diminui, linearmente, com a elevação da suplementação.

\section{AGRADECIMENTOS}

Os autores agradecem à empresa BÜNGE Alimentos, pelo fornecimento do alimento concentrado utilizado, ao Sr. José
Aloízio Fritzen pelos animais e pela infra-estrutura necessária para condução deste estudo e ao Centro Universitário Feevale, pelo apoio oferecido.

\section{REFERÊNCIAS BIBLIOGRÁFICAS}

MERTENS, D.R. Análise da fibra e sua utilização na avaliação de alimentos e formulação de rações. In: SIMPÓSIO INTERNACIONAL DE RUMINANTES, 1992, Lavras, MG. Anais... Lavras : SBZ, 1992. p.188219.

OLIVEIRA, M.V.M. et al. Avaliação da composição de cortes comerciais, componentes corporais e órgãos internos de cordeiros confinados e alimentados com dejetos de suínos. Revista Brasileira de Zootecnia, v.31, n.3, p.1459-1468, 2002.

OSÓRIO, J.C.S. Estudio de la calidad de canales comercializadas en el tipo ternasco según la procedencia: Bases para la mejora de dicha calidad en Brasil. 1992. 335f. Tese (Doutorado em Veterinária) Curso de Pós-graduação em Produção Animal, Universidad de Zaragoza.

OSÓRIO, J.C.S. et al. Produção de carne em ovinos de cinco genótipos: componentes do peso vivo. Ciência Rural, Santa Maria, v.26, n.3, p.471-475, 1996a.

OSÓRIO, J.C.S. et al. Componentes do peso vivo em cordeiros da raça Corriedale. In: REUNIÃO ANUAL DA SOCIEDADE BRASILEIRA DE ZOOTECNIA, 33., 1996, Fortaleza, CE. Anais... Fortaleza : Sociedade Brasileira de Zootecnia, 1996b. p.524-526.

OSÓRIO, J.C.S.; ASTIZ, C.S. Qualidade da carcaça e da carne ovina. In: ___ Programa de treinamento em ovinocultura. Porto Alegre, RS : FARSUL/SENAR, 1996. p.110-190.

SAS INSTITUTE. Sas User's guide: Statistics. Version 6.8. Cary, North Carolina, 1993. 956p.

SIQUEIRA, E.R. et al. Efeito do sexo e do peso ao abate sobre a produção de carne de cordeiro. Morfometria da carcaça, pesos dos cortes, composição tecidual e componentes não constituintes da carcaça. Revista Brasileira de Zootecnia, v.30, n.4, p.1299-1307, 2001. 